
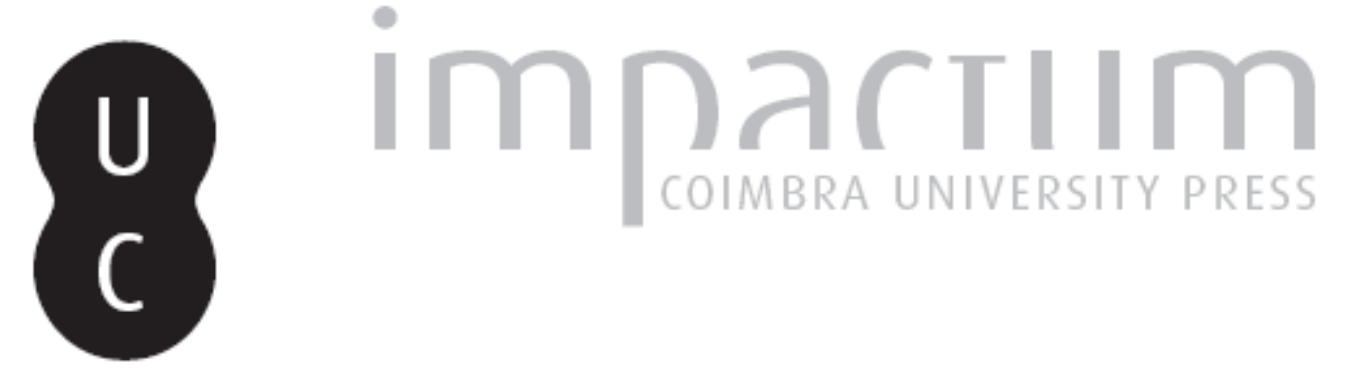

\title{
La Revolution Française dans la litterature portugaise: Felizmente Há Luar de Luís Sttau Monteiro
}

\author{
Autor(es): $\quad$ Freches, Claude-Henri
}

Publicado por: Imprensa da Universidade de Coimbra

URL persistente:

URI:http://hdl.handle.net/10316.2/43796

DOI:

DOI:https://doi.org/10.14195/2183-8925_10_37

Accessed : $\quad$ 26-Apr-2023 12:10:58

A navegação consulta e descarregamento dos títulos inseridos nas Bibliotecas Digitais UC Digitalis, UC Pombalina e UC Impactum, pressupõem a aceitação plena e sem reservas dos Termos e Condições de Uso destas Bibliotecas Digitais, disponíveis em https://digitalis.uc.pt/pt-pt/termos.

Conforme exposto nos referidos Termos e Condições de Uso, o descarregamento de títulos de acesso restrito requer uma licença válida de autorização devendo o utilizador aceder ao(s) documento(s) a partir de um endereço de IP da instituição detentora da supramencionada licença.

Ao utilizador é apenas permitido o descarregamento para uso pessoal, pelo que o emprego do(s) título(s) descarregado(s) para outro fim, designadamente comercial, carece de autorização do respetivo autor ou editor da obra.

Na medida em que todas as obras da UC Digitalis se encontram protegidas pelo Código do Direito de Autor e Direitos Conexos e demais legislação aplicável, toda a cópia, parcial ou total, deste documento, nos casos em que é legalmente admitida, deverá conter ou fazer-se acompanhar por este aviso.

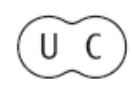


REVISTA DE HISTORIA DAS IDEIAS IO
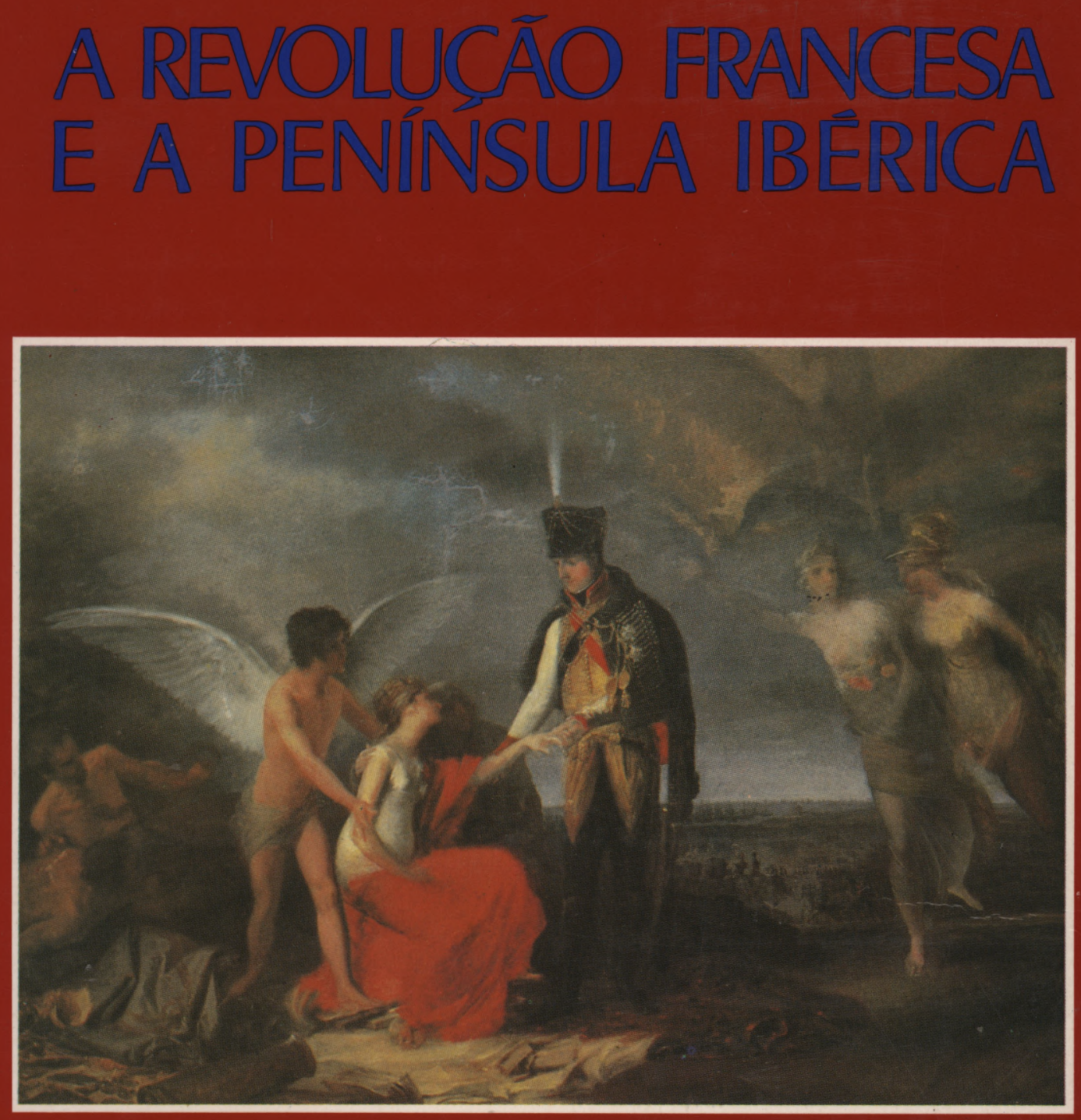

INSTITUTO DE HISTÖRIA E TEORIA DAS IDEIAS FACULDADE DE LETRAS 


\section{LA REVOLUTION FRANÇAISE DANS LA LITTERATURE PORTUGAISE: FELIZMENTE HA LUAR DE LUIS STTAU MONTEIRO}

En choisissant le Général Gomes Freire de Andrade pour personnage central, quoique absent, de son drame Felizmente há luar, publié en 1961 sous Salazar, Luís de Sttau Monteiro célébrait à sa façon l'immortelle Déclaration des Droits de l'Homme et du citoyen. Le texte en fut adopté le 26 août 1789. Gomes Freire avait suivi Napoléon en Russie. Or le tsar représentait aux yeux des fidèles de l'idéal révolutionnaire l'autocrate absolu. En revanche l'empereur, qui, lui-même, s'était rêvé Charlemagne, traînait dans ses campagnes la Révolution avec lui. «L'Empire, a-t-on écrit (1), fut le régime transitoire à travers lequel la Révolution s'enracina en France». Les guerres napoléoniennes ont dressé un barrage «que les défaites de 1814-1815 elles-mêmes n'ont pas pu bousculer, contre le reflux de l'ancien ordre social» $\left({ }^{2}\right)$. La commotion révolutionnaire, de l'avis de nombreux historiens, fut ainsi communiquée à presque toute l'Europe.

$\mathrm{Au}$ Portugal, soumis trois fois à l'invasion française, Gomes Freire, revenu en 1815 à Lisbonne, devenu grand maître de la Franc-Maçonnerie, était en quelque sorte le porteur de ce message de la Révolution française, dont l'Empereur, au milieu des vilénies de la guerre, éparpillait les échos. Ce héros fut d'emblée soupçonné d'être un meneur et un séide du libéralisme, tant il était plus ou moins adulé du populaire. Le Conseil de Régence le fit arrêter en 1815. Mais il ne put réunir les preuves pour le convaincre d'être à la tête de ceux qui espé-

* Université de Provence (Aix-Marseille I).

(1) Louis Bergeron, "L'Empire, 1719-1815», Histoire de la France par Georges Duby, vol. 2, p. 352.

(2) Ibidem. 
raient transformer le pays. Aussi l'Anglais Beresford s'en fut-il à Rio de Janeiro obtenir du roi les pleins pouvoirs. A son retour du Brésil, il fit appréhender le Général avec ses «complices». Enfermé dans le fort de S. Julião da Barra, Gomes Freire fut exécuté en mai 1817. Onze de ses partisans le furent au Campo de Santana, par pendaison, le 18 octobre. Gomes Freire était détenu dans la tourelle qui domine l'esplanade et que l'on aperçoit du large. Plus tard elle fut recouverte de briques blanches émaillées. Un portrait du prisonnier y gardait sa mémoire. Le Général mourut dans le champ voisin, à une centaine de mètres. Luís de Sttau Monteiro a mythifié la fin du chef présumé de la conspiration et celle des conjurés, dans le but de donner au drame un raccourci épique. La mort de Gomes Freire semble concomitante de celle des Onze. Le Campo de Santana, lieu d'exécution, rougeoie dans la nuit, au point que l'on se croit encore au temps de l'Inquisition. La présence chafouine du Principal - prélat associé à la Junta de Regência - dans Felizmente há luar, ne cesse d'évcquer l'anti-évangélisme de la hiérarchie et l'hypocrisie des pharisiens qui ont enlaidi l'histoire de l'Eglise. Homme de théâtre par instinct, l'auteur peut aussi bien revendiquer les libertés que Shakespeare prend avec l'Histoire, de même qu'il suit l'exemple de Brecht pour conter une histoire en diminuant les espaces et en syncopant les relais. Le héros n'est lui-même vu que par les autres: quelques gens du peuple, sa compagne, son ami. Il est hors de la scène, mais non du jeu, comme l'Arlésienne. En fait il symbolise la Révolution française. Elle-même n'est plus un événement historique, mais elle est un mythe développé en mystère où se célébrent la liberté, l'égalité, la possession des biens qui chassent la faim, le savoir qui protège de l'oppression. Pour l'auteur, ce qui reste des passions et des emportements ensanglantés de 89 à 1802, c'est en fait la Déclaration des Droits de l'Homme, instrument de libération et de progrès, conçu par les Français, mais valable pour tous les peuples.

La Déclaration des Droits de l'Homme met en avant, comme on le sait, quelques principes qui, aujourd'hui, nous paraissent évidents. L'article premier rappelle que «les hommes naissent et demeurent libres et égaux en droits». "Les distinctions sociales ne peuvent être fondées que sur l'utilité commune». Les droits de l'homme sont «la liberté, la propriété, la sûreté, et la résistance à l'opression». Cette liberté est limitée par celle d'autrui. «La loi est l'expression de la volonté générale: tous les citoyens ont droit de concourir personnellement ou par leurs représentants, à sa formation». Elle garantit l'égalité des citoyens qui sont «également admissibles à toutes dignités, 
places et emplois publics, selon leur capacité et sans autres distinctions que celles de leurs vertus et de leurs talents». La Déclaration garantit aussi contre les arrestations arbitraires. Elle interdit d'appliquer une loi avec effet rétroactif, quand il s'agit de punition. «Nul ne doit être inquiété pour ses opinions, même religieuses, ajoute-t-elle, pourvu que leur manifestation ne trouble pas l'ordre public établi par la loi». La liberté de penser et de communiquer, mais également de publier, est reconnue dans l'article XI, qui réserve le cas d'intervention de la loi en cas d'abu. L'article XIII prévoit la contribution fiscale de tous les citoyens pour assurer l'entretien do la force publique et pour les dépenses d'administration: «elle doit être également répartie entre les citoyens en raison de leurs facultés». Par eux-mêmes ou par leurs représentants, les citoyens constatent la nécessité des contributions. Ils en établissent l'assiette, le recouvrement et la durée. L'article XV, qui évoque la «dokimasie» des Grecs. vise les agents publics à qui la société a le droit de demander compte de son administration. La Déclaration prône la séparation des pouvoirs. Enfin l'article XVII garantit la propriété: droit inviolable et sacré, «nul ne peut en être privé, si ce n'est lorsque la nécessité publique, légalement constatée, l'exige évidemment, et sous la condition d'une juste et préalable indemnité».

Dans la pièce de Luís de Sttau Monteiro, apparaît la faim avec son corollaire, la mendicité. Pour le peuple, c'est la preuve absolue de l'inégalité. Il y a deux classes: ceux qui font l'aumône - bien chiche - et ceux qui la reçoivent. Le général Gomes Freire, lui-même, bien qu'il ne soit riche que de ses titres de guerre, ne saurait être considéré comme du peuple. Les yeux des «révoltés» ont beau voir en lui le chef de la Révolution possible, il a beau incarner, mythiquement, la Déclaration des droits de l'homme et du citoyen, dont il va être Ja martyr, il ne saurait être détaché de la caste supérieure. C'est pourquoi le peuple désespéré ne se dresse pas pour le délivrer. La seconde partie du drame, consacrée au fatum, ne peut que constater le triomphe de l'absolutisme appuyé sur le cynisme, la délation et la calomnie. Gomes Freire a été décrété ennemi public, on en a fait le chef de la révolte qui se préparait ou du moins qui provoquait certains borborygmes, et on l'a accablé sans preuves, le présumant coupable. Une affaire qui, aujourd'hui, préoccuperait Amnesty international.

$\mathrm{Si}$ le général Gomes Freire est un personnage symbole pour le peuple qui marcherait à ses côtés, s'il se donnait la peine de le soulever, son portrait est tracé par son ennemi, son «cousin», D. Miguel, le gouverneur qui le fera arrêter. 
«Notai, dit-il, que lhe não falta nada: é lúcido, é inteligente, é idolatrado pelo povo, é um soldado brilhante, é grão-mestre da Maçonaria e é, senhores, um estrangeirado». Franc-maçon, il a donc le culte du savoir qui garantit la liberté.

Le tableau social que fait deviner le premier acte veut sans doute rappeler, avec précaution, l'époque salazariste, dominée par le culte de l'argent. Tout et tout le monde sont à vendre. Car l'argent n'est pas seulement le moyen d'accéder à l'aisance, de sortir de la classe des mendiants: il fait accéder aux honneurs et aux charges. Cependant il pourrit tout. Beresford ne tient pas compte de son titre nobiliaire. En bon mercenaire, il lui faut conserver par tous les moyens, c'est à dire la liquidation d'un mouvement libéral qui ferait du Pcrtugal un pays politiquement comparable à sa patrie, l'Angleterre, sa solde de 16 contos par an. Quant aux défenseurs de l'absolutisme, D. Miguel et Sousa, ils semblent mûs par la défense de la monarchie de droit divin, la haine des cortes, par conséquent. En réalité, ils ne défendent que l'ordre traditionnel, lequel garantit les privilèges du clergé et de la noblesse. Pour eux le roi lui-même est au dessus de la loi. Sa présence est liée au messianisme et à l'événement mythique du Campo d'Ourique. Toute contamination française fait du sujet portugais un «estrangeirado», un suspect, susceptible de fomenter la Révolution. Certes des gens du peuple, fatigués des guerres napoléoniennes, ne veulent pas de la domination française (Charybde) sans vouloir pour autant passer sous le joug anglais (Sylla). En revanche, ils aspirent à l'égalité et à la liber té. Le Conseil de Régence a mis en place un système de mouchards dont la moitié surveille l'autre. Aussi bien ne faut-il pas s'étonner si, parmi eux, il se découvre des francs-maçons et des officiers chez qui se développaient les idées nouvelles: l'ambition et l'argent priment sur l'idéal. Le Pouvoir connaît bien leurs affiliations: quand ils vendent leurs renseignements. ils sont soumis au chantage afin qu'ils en disent plus. L'auteur dépeint une Lisbonne délatrice, qui est aussi celle de 1960. Les provocateurs y sont habiles. Par exemple Vicente, qui appartient a la dénonciation, démolit la stature héroïque de Gomes Freire en critiquant son inertie apparente: il est de l'autre classe, celle des oppresseurs. Il insiste sur sa qualité d' «estrangeirado", tablant sur le chauvinisme du peuple par ailleurs analphabète. Car l'«estrangeirado» a voyagé et il est instruit. Ce Vicente finira du reste comme chef de la police, tel le Vautrin de Balzac. Voyou cynique et pervers, mais intelligent et sournois, la délation le juchera sur un socle d'honorabilité. Paradoxalement, la Déclaration des droits de l'homme 
lui aura fourni le principe d'égalité. «E verdade que nasci aqui c. que a fome desta gente é a minha fome....», déclare-t-il. Puis il se dit condamné à la misère "par accident de naissance» pour conclure: «Que diferença há entre mim e um fidalgo qualquer?». Il prend donc le ton d'un révolutionnaire, mais il se range sous la bannière adverse, sachant que l'étiquette de traître est épargnée a qui gravit l'echelle sociale. Théâtralement, Vincent ressortit aux emplois du «bobo». Non qu'il soit ridiculo. Mais le cynisme, l'égoïsme, l'immoralité totale l'apparentent aux compagnons triviaux et vicieux des moines de comédias de santo. Luís de Sttau Monteiro insiste plusieurs fois sur la revanche du pauvre: faire l'aumône à qui la lui faisait naguère. C'est comme s'il rendait un soufflet. Vicente, irrespectueux à l'égard de l'autorité ecclésiastique, lance un trait révolutionnaire au Principal prélat, Sousa: le peuple ne s'intéresse qu'à calmer sa faim et non à ce qu'a écrit l'Ecclésiaste sur l'origine du pouvoir. Du reste, «si on lui apprenait à lire, il pourrait connaître l'Ecclésiaste». Ce qui lui vaut de la part de Sa Révérence un cri d'effroi: "a sabedoria é tão perigosa como a ignorância». Pour cet obscurantiste, la liberté est un mot dangereux. Le troisième est celui de «cortes», le parlement. Il l'est aussi pour D. Miguel. Celui-ci cherche ce qui peut être la cause de ce réveil révolutionnaire. Il songe aux événements du Pernambouc. On sait que le royaume du Brésil avait été établi le 17 décembre 1815. Il faisait partie d'un empire qui comprenait en outre le Portugal et les Algarves, sous le nom de Royaume uni. Or, le 6 mars 1817, le Pernambouc fait sécession, projette une constitution qui garantit la liberté de pensée, de presse et de religion. Elle est de plus anti-raciste. Mais cet essai de démocratie indépendante fut suffoqué. Sousa craint donc que cet événement tout frais ne trouble l'ordre au Portugal. Si la sottise caractérise le conservatisme du prélat, fidèle à l'alliance du trône et de l'autel, la roublardise et l'avidité imprégnent le style des mouchards de haut vol. Corvo rassure Morais Sarmento: on risque de les traiter de délateurs, craint celui-ci. Et Corvo de répliquer: l'honneur des enfants? «Ils porteront le nom de leur mère et dépenseront l'argent de leur père». L'achat de la respectabilité n'est pas si difficile avec une rente de 800 escudos par an. Le système mis en route pour faire croire à une révolte et désigner son chef ne diffère pas de celui de Don Basile: la calomnie, la rumeur, le «boato». Mais, tandis que D. Miguel et Sousa établissent la vilénie et récompensent la délation, Beresford oppose à ce petit pays où l'homme est opprimé, l'Angleterre libérale, «où les lois sont humaines, les personnes cultivées et la vie pleine de sens». 
Il proclame: "Sou duma terra onde um homem vive como um homem». Tel est le mépris du mercenaire anglais pour qui l'emploie. Il se conduit en colonisateur. Inconsciemment, il réfléchit certains aspects de la Révolution française. Par exemple, il oppose, dans son dialogue avec Sousa, le concept de liberté à la monarchie de droit divin. C'est pourtant au nom de ce principe que l'on bafoue gravement les Droits de l'homme. Gomes. Freire va être accusé sans preuves, il sera jugé en secret, et «pour éviter le pardon du roi, son exécution suivra immédiatement la sentence». L'émotion, au tribunal, remplacera la froide raison. Pour Sousa, il faut abattre la Franc-Maçonnerie. Pour D. Miguel, il est nécessaire d'arracher les plants d'anarchie et de jacobinisme, qu'il confond arbitrairement. Il définit alors son idéal conservateur: "Sonho com um Portugal próspero e feliz, com um povo simples, bom e confiante, que viva lavrando e defendendo a terra, com os olhos postos no Senhor». Il ne dit pas si ce peuple sera propriétaire de cette terre. Puis il rêve du deuxième état, une noblesse orgueilleuse, qui, de chez elle, dirigerait ce pays privilégié. Dans cette féodalité retrouvée, se situent un clergé, une noblesse et un peuple conscients de leur mission, intégrés dans la structure traditionnelle du Royaume. C'est dire qu'il soutiendra le miguélisme, la distinction des classes. Il est ennemi de l'opinion populaire et du vote universel. Aussi n'hésit-t-il pas à faire le portrait de l'homme adulé par le peuple, lequel, de ce fait, doit être le chef virtuel de la révolte: Gomes Freire d'Andrade est lucide, idolâtré du peuple; c'est un brillant soldat, le grand-maître de la Franc-Maçonnerie; de plus il est frotté de culture étrangère.

Cependant D. Miguel veut alerter l'opinion. Non que la Patrie soit en danger. Mais plutôt l'intérêt du Portugal, c'est à dire de tous. Dans leurs prêches, les moines, comme en France, à l'époque de la Sainte Ligue, stigamtiseront les ennemis de Dieu, fomenteurs des idées nouvelles. Les tambours battront l'alerte, pour créer une atmosphère d'épouvante. Les cloches sonneront le tocsin. Et soudain éclate un cri révolutionnaire, mais dans la bouche du chef du clergé: "A Pátria está em perigo». Et voici qu'il évoque l'immoralité des rebelles, le sac des maisons, le viol des femmes. Quant au gouverneur, il est disposé à sacrifier sa propre conscience et celle des autres, dans un ressaut de machiavélisme, sur l'autel de la Patrie.

On le voit, le vocabulaire est identique dans les deux parties, que l'on appartienne ou non aux tenants de la Révolution. L'intérêt joue de part et d'autre, et la soif de pouvoir. 89 a modifié les esprits, surtout en y semant la notion d'égalité de nature entre tous les hommes. Mais c'est surtout le droit 


\section{La Revolution Française dans la Litterature Portugaise}

à la possession, capable, croit-on, d'éteindre la faim, qui agite les Portugais de 1817 (et aussi de 1961), à en croire Luís de Sttau Monteiro. Toutefois, les malins estiment qu'ils ont plus de chances de passer dans le camp des nantis, en trahissant leur classe. Certes les demi-soldes et les vétérans des campagnes napoléoniennes sont encore prêts à se battre pour un régime libéral. Mais certains se situent à l'extrême-gauche, en quelque sorte, en faisant remarquer qu'un général, fût-il Gomes Freire, sera toujours du côté des nantis et des profiteurs, donc trahira inévitablement l'idéal de libération du peuple, à laquelle il avait prétendu se consacrer. Le tort de cette extrême-gauche est de penser, comme La Rochefoucault, que tout homme est mû par son propre intérêt et que l'altruisme n'existe pas.

La pièce est surtout basée sur les commentaires de ce qu'on peut appeler le Choeur, comme c'est le cas chez Eschyle. Rien ne peut arrêter le destin de Gomes Freire, ni les supplications de Mathilde, sa compagne, ni la conscience des $m \in m b r e s$ du Conseil de Régence. Au contraire ceux-ci ont décidé qu'il était le coupable: raison d'Etat, allégueront le Principal et Miguel. Quant à l'Anglais, peu lui chaud l'intégrité de l'accusé. Cet homme est l'ennemi idéal, taillé à la mesure de la stratégie qu'il imagine. On peut se demander: pourquoi l'auteur a-t-il voulu ressusciter les supplices de l'Inquisition? Pourquoi ces pendaisons, suivies d'incinératicn? Pourquoi ces cendres mêlées au Tage? En fait Sousa est comparable à l'évêque Cauchon et Gomes Freire à Jeanne d'Arc, la sainte patriote, mise en scène par Bernard Shaw. Mais surtcut, le bûcher imaginaire donne au titre son sens. A la fin de la pièce apparaît la Croix au pied de laquelle la compagne du martyr égrène son "pranto». Elle a revêtu sa robe verte. Là-dessus, s'allume un bûcher, tandis que la lune déploie sa lumière blanche. Et voilà que le drapeau de la République portugaise, sacralisé de la sorte, évoque l'espérance qui, l'épisode cruel passé, promet la liberté et l'amour, dans la vertu reconquise. Ce drapeau est le fruit du sacrifice de Gomes Freire, dont la sainteté a été proclamée, à la barbe du prélat félon et indigne de son titre de principal, par le petit moine qui a entendu la confession et les derniers propes de Gomes Freire.

Il y a d'ailleurs tcut un passage de la seconde partie, où le général incarne le Christ sauveur, condamné sur des allégations mensongères, supplicié, après avoir été vendu. Sans doute cela n'a-t-il pas été le cas pour le Portugais, encore que les délateurs aient été stipendiés. Mais Mathilde a reçu un sou d'aumône, transmis par un homme du peuple, qui a tendu la main au sortir d'un office. Elle jette la monnaie à Sousa, 
comme fit jadis Judas aux prêtres de Jérusalem. De plus, l'auteur, longuement, oppose, par la bouche de Mathilde, l'Eglise ccnservatrice au Christ qu'elle trahit constamment. Les atteintes aux Droits de l'homme sont dénoncés par l'ami Sousa Falcão: «Le Royaume est tombé aux mains de personnes viles, qui nomment âme l'estomac et qui élèvent des réglements de police à la classe des principes sacrés... Je vous avais bien dit de ne pas revenir! Mathilde: chaque fois que quelqu'un arrive de l'Etranger, les bases du Royaume sont ébranlées! Les rois du Rossio vivent dans la crainte de n'importe qui est capable de crier qu'ils vont nus». Comme dans toute dictature la Cité a priorité sur le Droit des personnes. Comme dit Beresford, «la seule existence de certains hommes est d'emblée un crime». Beresford finit par laisser entendre qu'on va juger le jeune homme et qu'on le tuera. Alors Manuel oppose le peuple avili par la réaction, réclamé par qui a besoin d'aide, à ceux qui auront besoin de son aide. Mais, avili, mendiant sa pitance, il ne peut alléguer que la servitude due à l'ignorance et ne fournira point à l'accusé que l'ombre des misérables.

Toutefois Mathilde apprend qu'on a défendu au «coupable» de se choisir un avocat. Il en va de même pour les douze autres. Refoulée par le Gouverneur, elle veut faire intervenir le prélat Sousa. Or, tandis que ce dernier la laisse approcher, ses termes décrivent le Portugais comme un saint. C'est ce que confirme le moine confesseur de l'accusé. Ainsi le bas-clergé prend-il en charge le franc-maçon dont se débarrasse le gouvernement. Il l'admire, essaie, vainement, d'infléchir son destin. Le dialogue fait place au soliloque de la jeune femme, à jamais vouée à la solitude. Dans la dernière vision du supplice où meurt le soldat couvert de gloire en Europe, elle prie afin que le monde, entr'ouvert, conserve au Portugal ses emblèmes.

Ainsi le vêtement de la jeune femme devient-il la couleur saisissante, entrée dans le symbolisme qui frappe le Portugal de 1961. C'est le vert. Associé au blanc du rêve et au rouge des braises, voilà recomposés les coloris devant lesquels s'anéantira, pour revivre plus de cent ans après, la race de Gomes Freire d'Andrade.

Donc Felizmente há luar souhaite que chaque lever de lune ressuscite chez le patriote portugais le souvenir de Gomes Freire, héros perdu et sauvé. 